\title{
Microbial community structure and distribution in the air of a powdered infant formula factory based on cultivation and high-throughput sequence methods
}

\author{
Shuang Wu, ${ }^{* 1}$ Yujun Jiang, ${ }^{* 1}$ Binbin Lou, ${ }^{*}$ Jing Feng, ${ }^{*}$ Yanhong Zhou, ${ }^{*}$ Ling Guo, ${ }^{*}$ Stephen J. Forsythe, $†$ \\ and Chaoxin Man*2 \\ *Key Laboratory of Dairy Science, Ministry of Education, Department of Food Science, Northeast Agricultural University, Harbin, China, 150030 \\ †Foodmicrobe.com, Nottingham, United Kingdom, NG12 5GY
}

\begin{abstract}
The air in a powdered infant formula (PIF) factory is a potential transfer medium for microorganisms. In this study, air samples from 6 main processing areas, almost covering the whole PIF processing line and 1 outdoor location, were collected from a PIF manufacturing plant during the winter and summer periods. A cultivation-based and an Illumina (San Diego, CA) high-throughput 16S rRNA sequencing method was used to investigate the community structures and distributions of bacteria in the air. High microbial diversity (25 genera, 56 species), with a dominant community including Staphylococcus, Bacillus, Acinetobacter, and Kocuria, was found by the cultivation-based method. Moreover, 104 genera were obtained from all samples by high-throughput sequencing methods. Lactococcus (32.3\%), Bacillus (29.6\%), and Staphylococcus (14.0\%) were the preponderant genera. The indices from highthroughput sequencing results indicated that the bacterial community of the air samples was highly diverse. Significant differences in the diversity and distribution at 6 sampling locations were revealed using the 2 methods. In particular, the packaging process contained the highest proportion $(39.4 \%)$ of isolated strains. The highest diversity in bacterial community structure was found in the outdoor location. More bacterial isolates and higher community diversity were observed in the summer samples compared with the winter samples. In addition, some pathogens, such as Acinetobacter baumannii, Bacillus cereus, and Staphylococcus cohnii, were mainly found in the large bag filling process, can filling, and packaging process areas. The present study provides greater insight into the microbial community and identifies potential sources of air contamination in PIF production environments and can serve as a guide
\end{abstract}

Received October 10, 2017.

Accepted March 24, 2018.

${ }^{1}$ Both authors contributed equally to this paper.

${ }^{2}$ Corresponding author: mcxwh2006@qq.com to reduce the risk of microbial contamination in the production of PIF.

Key words: airborne microorganism, bacterial diversity, powdered infant formula, milk, highthroughput sequencing

\section{INTRODUCTION}

Powdered infant formula (PIF) is an effective breast milk substitute, providing all or most of the nutritional requirements for the normal growth and development of infants (Kent et al., 2015). However, PIF is not considered to be a sterile product, which has caused a potential safety risk to infants' health (Mullane et al., 2007, 2008). Cronobacter spp. (Enterobacter sakazakii), an important potential pathogen for neonates and infants, has been detected in PIF, dried baby foods, milk powder, and the production environment of PIF (Iversen and Forsythe, 2004; Sonbol et al., 2013; Fei et al., 2015). In a study conducted in Malaysia, it was reported that $13 \%$ of PIF, follow-up formulas, and infant foods samples $(\mathrm{n}=90)$ had viable counts higher than $10^{4} \mathrm{cfu} / \mathrm{g}$. Aerobic plate counts of PIF samples ranged between $10^{2}$ and $7.3 \times 10^{3} \mathrm{cfu} / \mathrm{g}$. The most frequent Enterobacteriaceae isolated was Enterobacter spp., followed by Citrobacter spp. and Klebsiella spp. (Abdullah Sani et al., 2013). Various methods have been developed for the detection and identification of microbial hazards in infant foods, including real-time PCR (Fricker-Feer et al., 2011), probe-magnetic beads (Xu et al., 2014), and matrix-assisted laser desorption/ionization timeof-flight MS ( $\mathrm{Lu}$ et al., 2014). Aflatoxin $\mathrm{M}_{1}$, a carcinogenic mycotoxin, has also been found in infant food (Kabak, 2012). The presence and levels of aflatoxin $\mathrm{M}_{1}$ contamination were investigated in 34 infant formula samples, with only 1 positive sample detected at a concentration of $0.0061 \mu \mathrm{g} / \mathrm{kg}$ (Er et al., 2014). Besides the risk of intrinsic microbial contaminants, poor hygienic practices and the use of inadequately sanitized utensils can cause the extrinsic contamination by pathogenic bacteria during PIF reconstitution (Araújo et al., 2015; 
Rachon et al., 2017). Therefore, reducing the microbial contamination of PIF is of considerable importance to neonatal health.

In the food manufacturing environment, air can be considered to be a transfer medium for the dissemination of bioaerosols, with airborne microorganisms including bacteria, fungi, viruses, pollen, and microscopic protozoa (Stetzenbach et al., 2004; Lee, 2011). Air contamination through poor environmental conditions can enable pathogens to be spread widely with air currents (Eduard et al., 2012). Consequently, airborne pathogens may be in contact with final products, equipment surfaces, and containers and result in PIF contamination during the production process (Brandl et al., 2014). Bacteria associated with PIF contamination, especially Enterobacteriaceae, have been isolated from the air of PIF processing environments (Angulo et al., 2008; Jacobs et al., 2011). Previous studies have proposed that the food-borne pathogenic bacteria suspended in the air could cause severe neonatal infections through contamination of the final products (Proudy et al., 2008; Yan et al., 2012). Jacobs et al. (2011) reported the high recovery of the neonatal pathogen Cronobacter from airborne dust collected by the air filters. The volume of dust was considerable and had been fed back into the production line. Thus, airborne bacteria are regarded as a potential contamination source in PIF processing and may result in product quality and safety issues.

Brandl et al. (2014) demonstrated the distribution of microorganisms in the air of a Swiss milk processing facility using the traditional cultivation-based method. Ninety-four morphologically different colony types were identified from the air of different sampling locations. These isolates included opportunistic pathogens, such as Roseomonas mucosa, Bacillus cereus, Brevundimonas diminuta, Moraxella osloensis, Macrococcus caseolyticus, Staphylococcus cohnii, Staphylococcus epidermidis, and Staphylococcus saprophyticus. Most of the opportunistic pathogens were isolated from the processes of tin filling and bag filling. However, not all the opportunistic pathogens can be cultured. In previous studies, the majority of airborne microorganisms were unculturable, with only 0.01 to $10.0 \%$ of microorganisms able to be detected by cultivation-based methods (Horner-Devine et al., 2004; Riesenfeld et al., 2004). Consequently, the structure of microbial communities in the air cannot be completely determined using conventional cultivation methods. To overcome this shortcoming, high-throughput sequencing technology can be used to provide more detailed information concerning microbial community structures. At present, this technology has been used to analyze environmental biodiversity in many fields, such as wastewater treatment and soil (Roesch et al., 2007; $\mathrm{Hu}$ et al., 2012; Poulsen et al., 2013). Moreover, the microbial community composition of the air of a PIF factory is expected to be highly diverse. Therefore, determining the airborne microbial community structure within a PIF factory is warranted.

In this study, air samples were collected from 6 locations (5 indoor locations and 1 outdoor location) during winter and summer periods. These covered the entire PIF processing line. The microbial community structures of the air samples obtained from a typical PIF factory were investigated using both cultivationbased and Illumina (San Diego, CA) high-throughput sequencing methods. Our study aimed to provide a more comprehensive understanding of the microbial community structure in the air of a PIF factory, which is of relevance to microbial source tracking of air contamination during the production process.

\section{MATERIALS AND METHODS}

\section{Sampling Locations}

This study was conducted in a typical wet-processing PIF factory located in the northeast of China. A flowchart of the PIF process is shown in Figure 1. Six sampling locations $(\mathrm{A}-\mathrm{F})$ were selected in this study. Indoor sampling locations covered the whole processing line, including pretreatment (A), production (B), large bag filling (C), can filling (D), and packaging (E). An outdoor sampling location (F) was used as the reference.

\section{Sample Collection}

The air samples were collected at each sampling site during the same period between 0800 and $1700 \mathrm{~h}$ in 2 different seasons, winter (November to December 2014) and summer (June to July 2015). A JWL-SW1 microbe air sampler (Sennon, Beijing, China) with a flow rate of $200 \mathrm{~L} / \mathrm{min}$ was used for collecting airborne bacteria. During each sampling, the sampler was mounted on a tripod at a height of about $1 \mathrm{~m}$ above the floor. To reduce environmental contamination, before sampling the impactor was disassembled and irradiated with UV radiation for $1 \mathrm{~h}$. Between each sampling, the disc in contact with air and the surface of the sampler were wiped with $75 \%$ ( $\mathrm{vol} / \mathrm{vol}$ ) ethanol solution for $3 \mathrm{~min}$ (Wang et al., 2010; Leung et al., 2014). The impactor was sealed when not in use.

Cultivable samples were collected on 90-mm trypticase soy agar (TSA) plates with 3 repetitions for each time point. Sampling time was $2.5 \mathrm{~min}$, and impaction volume was $500 \mathrm{~L}$. The samples were immediately incubated at $37^{\circ} \mathrm{C}$ for 3 to $7 \mathrm{~d}$ after collection (Zollinger et al., 2006). The amount of sampling was dependent 
on the space and area of each sampling location. Therefore, a total of 6 samples were collected in each season, including 144 plates in the pretreatment area (A), 216 plates in the production area (B), 162 plates in the large bag filling area (C), 180 plates in the can filling area (D), 216 plates in the packaging area (E), and 144 plates in the outdoor sampling location $(\mathrm{F})$. Based on different colony morphologies, the bacterial colonies were isolated for further analysis.

The air sampler was fitted with a polycarbonate membrane filter (Mili Membrane Separation Technology Co. Ltd., Shanghai, China) to collect the air samples for Illumina high-throughput sequencing. At each sampling location, the air sample was collected for about $2 \mathrm{~h}$ at a flow rate of $200 \mathrm{~L} / \mathrm{min}$ in triplicate. A total of 12 samples were collected from 6 sampling locations
(A-F) during summer and winter. After sampling, the filters were placed in $50-\mathrm{mL}$ sterile centrifuge tubes and immediately stored at $-80^{\circ} \mathrm{C}$ until further treatment.

\section{DNA Extraction and PCR Amplification}

The DNA of each single colony from the TSA plates were extracted using a TIANamp bacterial DNA extraction kit (Tiangen Biotech, Beijing, China) according to the manufacturer's instructions and stored at $-20^{\circ} \mathrm{C}$ until required. The DNA was amplified using $16 \mathrm{~S}$ rRNA universal primers, and the PCR amplification was performed according to previous reports (Guo et al., 2014; Li et al., 2015). The PCR products were visualized by $1.0 \%$ agarose gel electrophoresis after staining with ethidium bromide.

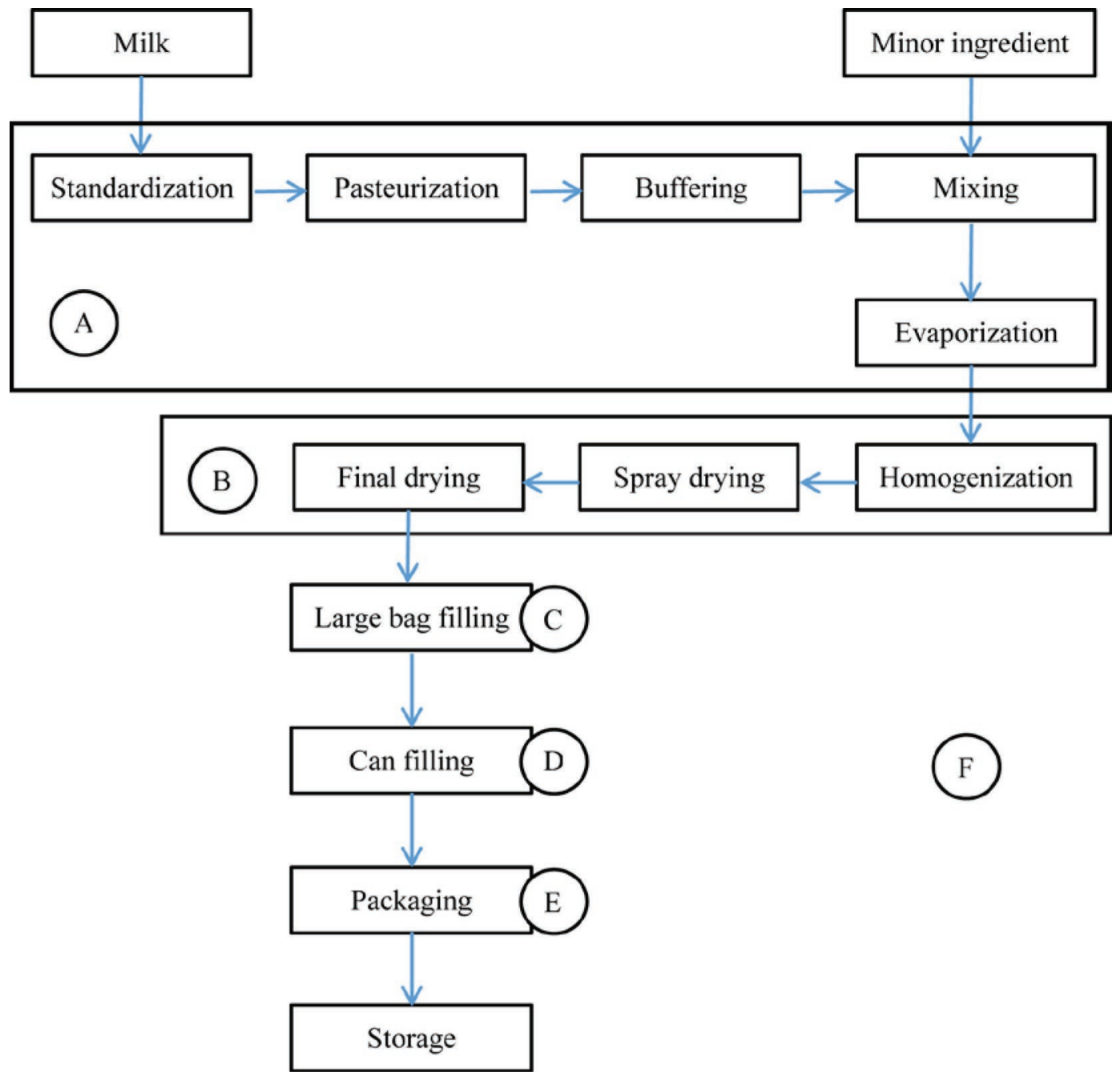

Figure 1. Flowchart of powdered infant formula production. Indoor sampling locations covered the whole processing line, including pretreatment (A), production (B), large bag filling (C), can filling (D), and packaging (E). An outdoor sampling location (F) was used as the reference. Color version available online. 
Each polycarbonate membrane filter was cut into small pieces and put into $15-\mathrm{mL}$ sterile centrifuge tubes with sterilized PBS. After gentle vortexing at $4^{\circ} \mathrm{C}$ and centrifugation at $300 \times g$ for $1 \mathrm{~h}$, the suspension was used to extract DNA according to the manufacturer's instructions for the Power Soil DNA extraction kit (MoBio Laboratories, Carlsbad, CA). The concentration and purity of each extracted DNA were determined by microspectrophotometry (NanoDrop ND-1000, Thermo Fisher Scientific, Waltham, MA). The concentration of DNA was about $10 \mathrm{ng} / \mu \mathrm{L}$. The range of optical density $260 / 280$ was 1.84 to 2.01 , and the range of optical density $260 / 230$ was 2.14 to 2.30 . The DNA with the V4-V5 hypervariable regions of the bacterial $16 \mathrm{~S}$ rRNA gene was amplified using the universal primer $515 \mathrm{~F}$ (5'-GTGCCAGCMGCCGCGG-3') and 907R (5'-CCGTCAAATTCMTTTRAGTTT-3'). A barcode was incorporated with the 5 '-end primer, and PCR assays were performed as described by Tian et al. (2015). All PCR products of each sample were purified using a PCR clean-up kit (TaKaRa, Dalian, China).

\section{High-Throughput Sequencing and Data Analysis}

The purified PCR products were $30 \mathrm{ng} / \mu \mathrm{L}$ and sequenced on an Illumina Miseq PE250 sequencer by Majorbio Biopharm Technology Co. Ltd. (Shanghai, China). The raw sequences generated from different samples were assigned by referring to the Ribosomal Database Project (http://rdp.cme.msu.edu/; Wang et al., 2007) to trim off the adapters and barcodes to reduce sequencing errors. Sequences longer than $200 \mathrm{bp}$ in length with a sequencing quality value greater than 25 were used in the subsequent analyses. Chimeras and contaminants were identified and discarded to improve sequence quality by Mothur (version 1.29.2; Schloss et al., 2009). The remaining sequences of each sample were used to define the operational taxonomic units (OTU) based on $97 \%$ sequence similarity level (Miao et al., 2015). The representative sequences, selected from the most abundant sequence in each OTU at the same sequencing depth, were classified by Ribosomal Database Project naive Bayesian classifier with a confidence threshold of $80 \%$ (Wang et al., 2007). The relative abundance of each sample was set as the number of sequences of a given phylogenetic group divided by the total number of target genera sequences.

\section{Statistical Analysis}

The 16S rRNA sequences were identified by the BLASTN algorithm in the GenBank database (http:// www.ncbi.nlm.nih.gov/BLAST/). The diversity and richness indices based on OTU were calculated to de- scribe the bacterial community of each sample. For alpha diversity analysis, Chao1 estimates the amount of OTU in each sample and the species abundance (Chao, 1984), abundance-based coverage estimator (ACE) is used to estimate the community richness (Eckburg et al., 2005), and Shannon index reveals the microbial diversity in samples (Washington, 1984); microbial diversity is higher with the increase of Shannon index (Zhang et al., 2016). For beta diversity analysis, the principal component analyses were performed to explore the relationship between microbial composition and samples with R (version 3.0.2; R Core Team, 2014). The input data set consisted of a $12 \times 104$ matrix based on the relative abundance OTU of different samples at the genus level. The rows represented 12 air samples collected in different seasons and sampling sites, and the columns represented the relative abundance of OTU about 104 genera. Meanwhile, the analysis of Duncan's multiple range tests was used to compare the diversity and richness indices of different samples by SPSS 20.0 software (SPSS Inc., Chicago, IL).

\section{Nucleotide Sequence Accession Numbers}

The bacterial nucleotide sequences isolated from this study were deposited in GenBank under accession numbers KU922128 to KU922528.

\section{RESULTS AND DISCUSSION}

\section{Microbial Community Structure of Culturable Microbes}

Structure of Bacterial Community in All Samples. A total of 401 morphologically different bacterial colonies (108 colonies from the winter samples and 293 colonies from the summer samples) were isolated using the cultivation-based method. Most isolates (92.02\%) were gram-positive organisms. Based on the highest similarity of sequences by using BLAST in NCBI, the bacterial isolates were assigned to 4 bacterial phyla: Firmicutes (82.79\%), Actinobacteria (9.23\%), Proteobacteria (6.73\%), and Bacteroidetes (1.25\%). All the isolates belonging to these 4 phyla were further classified into 24 genera and 56 species. Staphylococcus, Bacillus, Acinetobacter, Kocuria, and Aerococcus were considered to be the prominent genera, with relative proportions of 39.90, 35.41, 5.24, 3.24, and 3.00\%. Meanwhile, S. epidermidis (13.97\%) and Bacillus licheniformis (12.97\%) were the dominant species across all the sampling locations. In addition, Staphylococcus hominis (7.73\%), B. cereus $(7.23 \%)$, and S. cohnii $(4.48 \%)$ were found as the primary species (Supplemental Table S1; https:// doi.org/10.3168/jds.2017-13968). Brandl et al. (2014) 
reported that the airborne microorganisms were distributed among 4 phyla (Firmicutes, Actinobacteria, Proteobacteria, and Bacteroidetes) in a Swiss dairy plant. Furthermore, 25 bacterial genera and 58 species were observed, with the majority being gram-positive genera Staphylococcus and Bacillus. Hence, the community composition and structure using a cultivationbased method in this study were similar to the results previously obtained by Brandl et al. (2014).

According to the risk assessment meeting on E. sakazakii and other microorganisms in PIF by FAO/WHO (2004), there are 3 categories of bacteria of concern in PIF: categories A, B, and C. Salmonella serovars and Cronobacter species are designated category $\mathrm{A}$ as there was clear evidence of causing neonatal infections through the consumption of contaminated PIF. Some pathogenic bacteria found in our study, such as B. cereus and Staphylococcus aureus, belong to category C, in which the connection with causing neonatal infection through contaminated PIF was less plausible than in categories $\mathrm{A}$ and $\mathrm{B}$ or not yet demonstrated (FAO/ WHO, 2004). Bacillus cereus can produce enterotoxins or cereulide and can cause food poisoning, emesis, and infection diseases (Stenfors Arnesen et al., 2008). More seriously, it can produce endospores that survive in extreme conditions for a long time (Michaelsen et al., 2010). Staphylococcus aureus, one of the most important species related to food poisoning, was mainly isolated from the locations of can filling and packaging by the culture method. Other pathogenic microorganisms recovered in this study were Acinetobacter spp., including Acinetobacter baumannii, Acinetobacter calcoaceticus, and Acinetobacter lwoffii. Acinetobacter spp. were mainly found in pretreatment and packaging locations during summer sampling in our study (Table 1). Acinetobacter spp. are in category B. These are organisms with plausible but undemonstrated connection with causing neonatal infection through contaminated PIF. This category also includes various members of the Enterobacteriaceae. Hence, the persistence of Acinetobacter species in desiccated PIF could be a risk to neonatal health (Juma et al., 2016). Once the air of a PIF production environment is contaminated with a high concentration of pathogens, these bacteria may have an opportunity to contaminate the equipment surfaces and final products. Thus, the concentration of airborne pathogenic microorganisms should be monitored and the transfer of airborne bacteria into processing areas should be highly controlled to reduce contamination of the airborne pathogens in the production of PIF.

Distribution of Bacteria at Each Sampling Site. A different distribution of bacteria could be observed in the 6 sampling sites using the cultivationbased method. The site with the highest diversity of species was the packaging process area ( 47 species, 158 colonies), followed by can filling area (20 species, 70 colonies), large bag filling area (20 species, 38 colonies), pretreatment process area (18 species, 38 colonies), production process area (18 species, 51 colonies), and outdoor location (15 species, 46 colonies). Among these sampling sites, S. epidermidis and B. licheniformis were isolated from all the sites, especially the production and packaging area. Micrococcus luteus and S. aureus were mainly found in the can filling process. The packaging site was the primary separating site of $B$. cereus strains compared with other sampling sites. The majority of A. baumannii strains were isolated from the packaging location. Acinetobacter calcoaceticus was isolated only in pretreatment and large bag filling locations, and M. osloensis and Staphylococcus caprae were obtained only from the packaging area. Neisseria flavescens was isolated only from the pretreatment site. Similarly, Brevibacterium frigoritolerans and Dermacoccus nishinomiyaensis were identified only in the process of can filling. Additionally, the bacterial isolates Macrococcus caseolyticus and Kocuria erythromyxa were detected in a Swiss milk processing environment (Brandl et al., 2014) but were not found in our study. This might be due to the differences of geographical regions, seasons, and other environmental factors.

In our study, most strains were collected from the packaging process area (E), followed by the large bag filling area (C) and bag or tin filling locations (D); these were probably related to more frequent human movement around these production areas. Studies have indicated that microorganisms were present in the shoes of employees, external roofs above spray driers, tanker bay, powder-packaging rooms, air treatment areas, and the areas with high foot traffic in the milk powder factories, leading to cross-contamination into spray-drying facilities and the entry of pathogens into products (Craven et al., 2010; Sonbol et al., 2013). Therefore, the staff assignments should be rational and strict hygiene standards for workers must be implemented to reduce personnel movement and the risk of microorganisms spreading in the clean work area.

Seasonal Distribution of Bacteria at the Species Level. According to the cultivation-based method, about 18 genera ( 36 species) and 20 genera (46 species) were found in the winter samples and summer samples, respectively. The results indicated that the bacteria collected from summer samples had a higher diversity compared with those collected from winter samples. Some differences in bacterial distribution (shown in Table 1) were revealed at 6 sampling locations during both winter and summer sampling. For example, $M$. luteus accounted for $4.63 \%$ of the total isolates collected from winter samples, which is higher than that 
Table 1. Distribution of culturable bacteria in the air of a powdered infant formula factory at 6 sampling locations $\left(\mathrm{A}-\mathrm{F}^{1}\right)$ during winter and summer

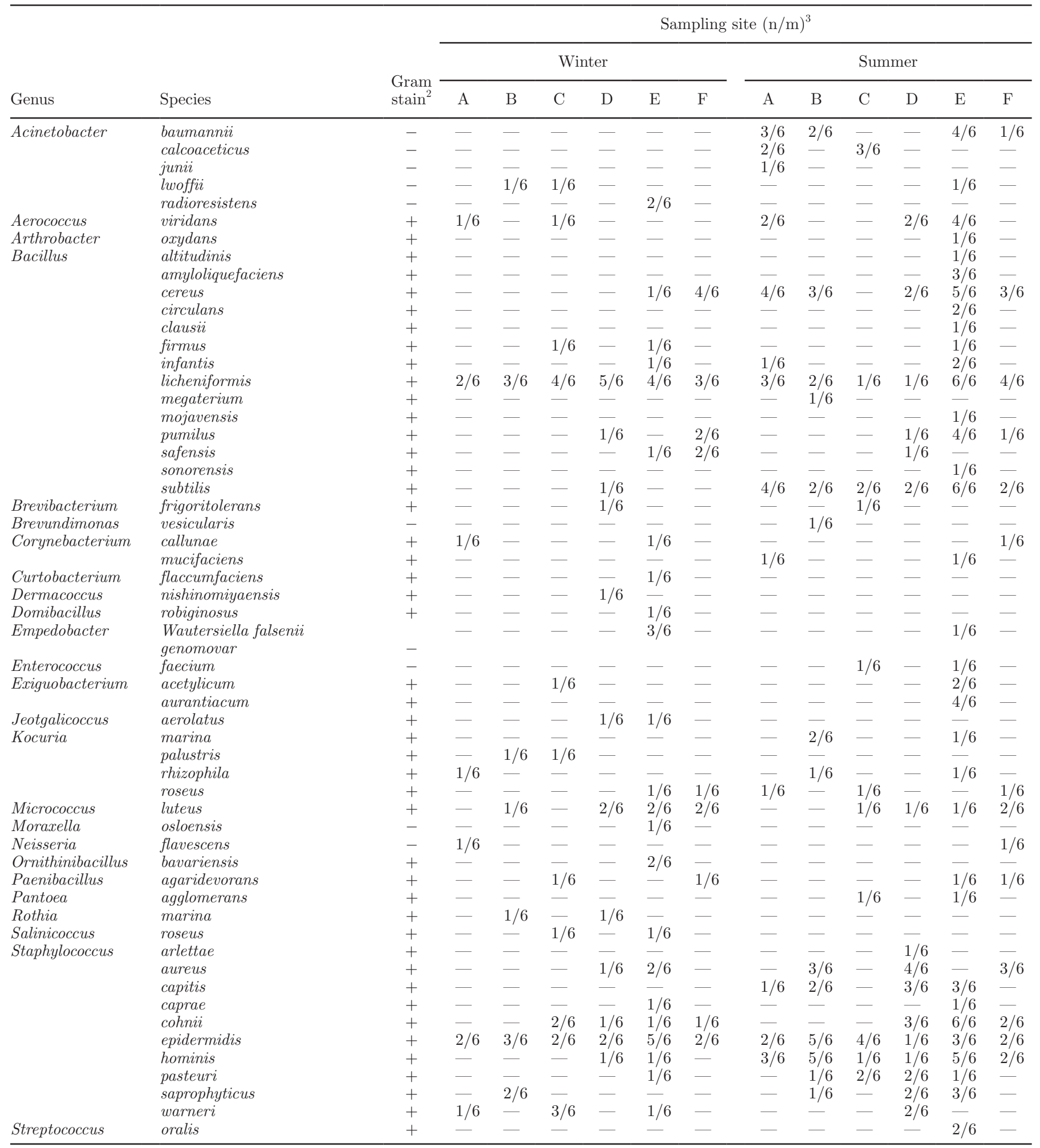

${ }^{1}$ Indoor sampling locations covered the whole processing line, including pretreatment (A), production (B), large bag filling (C), can filling (D), and packaging (E). An outdoor sampling location (F) was used as the reference.

${ }^{2}-=$ negative; $+=$ positive.

${ }^{3} \mathrm{n}=$ the number of times each species was isolated in each location; $\mathrm{m}=$ the total number of times for sampling. Dash indicates $\mathrm{n}=0$. 
in summer $(2.05 \%)$. In contrast, the number of Bacillus subtilis was significantly higher $(P<0.05)$ in summer $(9.22 \%)$ than in winter $(1.85 \%)$, and a similar trend was obtained with B. cereus, S. aureus, and S. hominis. The distribution of bacterial species with low abundances varied according to the different seasons. Acinetobacter radioresistens, Jeotgalicoccus aerolatus, Moraxella osloensis, and Salinicoccus roseus were found only in the winter samples, whereas A. baumannii, A. calcoaceticus, Acinetobacter junii, Brevundimonas vesicularis, Rothia marina, and S. caprae were detected only in the summer samples.

\section{Microbial Diversity by High-Throughput Sequencing Technique}

Bacterial Diversity of Each Sample. The bacterial diversity of air samples from 6 locations of a PIF factory in summer and winter was assessed by the Illumina high-throughput sequencing technique. A total of 245,729 sequences were obtained from air samples. Following quality controlling, 19,306 high-quality sequences were selected from each sample. As shown in Table 2, the coverage rate of each sample was more than 0.97 , suggesting that the number of sequences was sufficient to analyze the bacterial community diversity. A series of metrics that displayed the bacterial diversity of air samples, including OTU, the Shannon diversity index, ACE, and Chao1 richness estimator, were calculated and compared (Table 2). A total of 140 bacterial OUT were identified with $97 \%$ sequence similarity. The number of OTU from the outdoor sample were higher than all the indoor samples in both winter $(65 \pm 5)$ and summer $(84 \pm 7)$. There was no difference $(P<$ $0.05)$ between the 5 main processing stages of the PIF factory except for packaging site. However, OTU of each sample in summer were obviously higher $(P<$ 0.05) than in winter, which was further confirmed by the rarefaction curve (Supplemental Figure S1; https:/ / doi.org/10.3168/jds.2017-13968). For the other metrics (Shannon, ACE, and Chao1), no statistically significant differences were observed in the indoor air samples collected from the pretreatment site (A), production site (B), can filling site (D), and packaging site (E). Exceptionally, the sample from the large bag filling site (C) had differences $(P<0.05)$ in richness for Chaol $(84.50$ \pm 7.31 ), suggesting that the differences in the indoor sampling sites might have no obvious effect on bacterial diversity. The greater diversity index (Shannon) and richness indices (ACE and Chao 1) showed that the outdoor samples had a relatively higher bacterial diversity compared with indoor samples. Statistical differences $(P<0.05)$ among the metrics of each sampling sites indicated that the season might have an effect on the diversity of bacterial community structures between winter and summer sampling.

Bacterial Community Structures of All Samples. The sequences were identified at the phylum and genus levels by high-throughput sequencing technology. About $90 \%$ of the total bacterial sequences belonged to Firmicutes, Proteobacteria, Actinobacteria, and Bacteroidetes, and the remaining sequences belonged to 12 minor phyla, including Chlorobi, Chloroflexi, and Deferribacteres. The bacterial community structures of air samples from different sites in summer and winter are shown in Figure 2. In total, 104 different genera

Table 2. Comparison of the estimated operational taxonomic units (OTU), richness, and diversity indices of each sample collected from 6 sampling locations $\left(\mathrm{A}-\mathrm{F}^{1}\right)$ both during winter and summer $( \pm \mathrm{SD})$

Sampling location

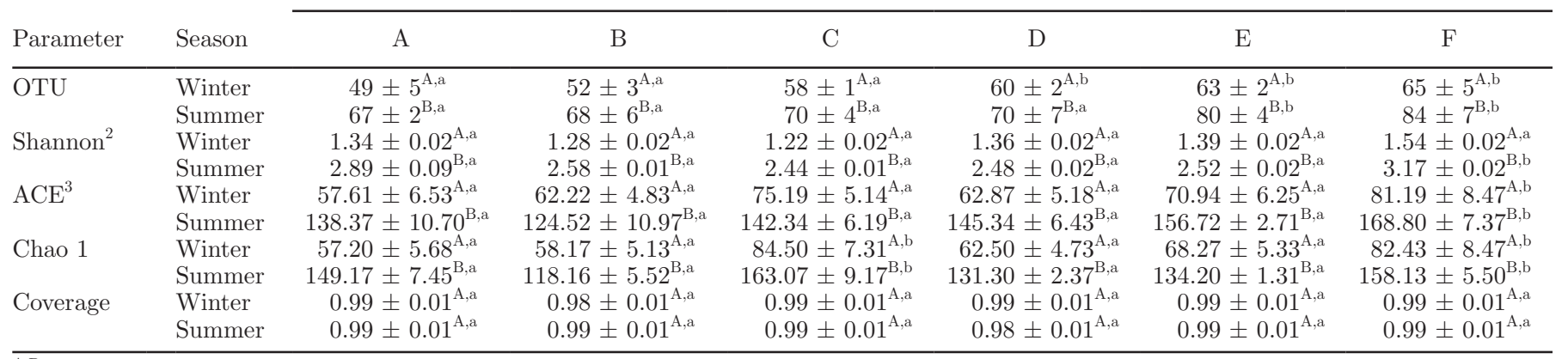

\footnotetext{
${ }_{\mathrm{A}, \mathrm{B}}$ Means within a column with different superscripts are significantly different $(P<0.05)$.

${ }^{\mathrm{a}, \mathrm{b}}$ Means within a row with different superscripts are significantly different $(P<0.05)$.

${ }^{1}$ Indoor sampling locations covered the whole processing line, including pretreatment (A), production (B), large bag filling (C), can filling (D), and packaging (E). An outdoor sampling location (F) was used as the reference.

${ }^{2} \mathrm{~A}$ higher number represents more diversity.

${ }^{3}$ Abundance-based coverage estimator.
} 

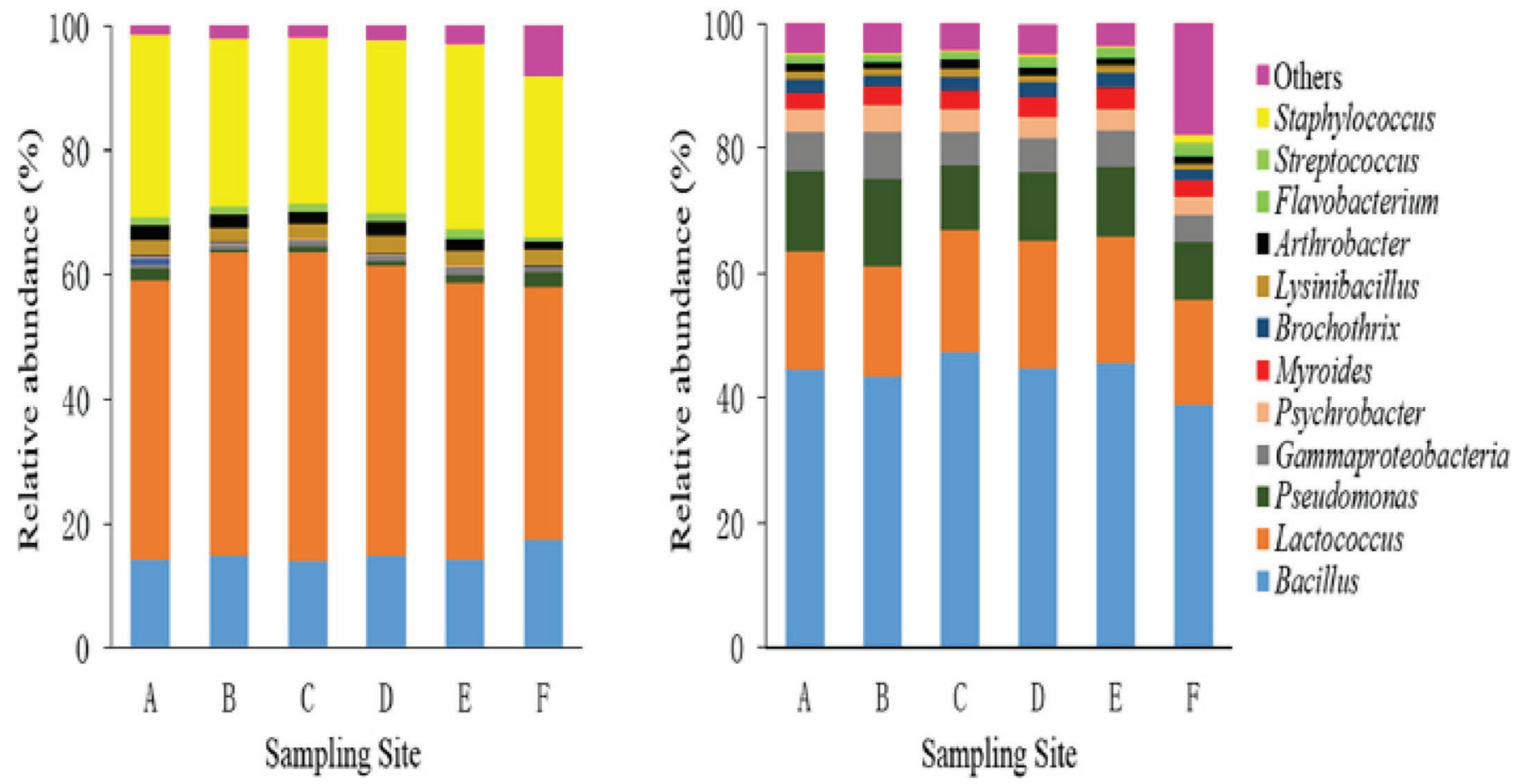

Figure 2. Relative abundances of the bacterial genera at 6 sampling locations in the winter (panel A) and summer (panel B) based on highthroughput sequence methods. Indoor sampling locations covered the whole processing line, including pretreatment (A), production (B), large bag filling (C), can filling (D), and packaging (E). An outdoor sampling location (F) was used as the reference. Color version available online.

were identified by high-throughput sequencing method, and the number of genera was much higher than that detected by culture-based method. In the same season, the bacterial community structures of different samples were approximately similar at the genus level. During both winter and summer sampling, Bacillus, Lactococcus, Staphylococcus, and Pseudomonas were the dominant genera. They were widely distributed and known as common components of environment, accounting for $70 \%$ of the obtained bacterial communities. Firmicutes, which the genera Bacillus, Lactococcus, and Staphylococcus belong to, was the dominant phyla detected in our study.

However, the relative abundance of each genus differed among the 6 sampling locations. For instance, during winter outdoor sampling, the proportion of Staphylococcus and Lactococcus was the smallest compared with indoor sites, whereas the proportion of Bacillus was the largest. In the summer outdoor samples, the percentage of Bacillus, Lactococcus, and Pseudomonas was the smallest compared with the indoor samples. In this study, other genera with average relative abundances $<0.01 \%$ were assigned in the same group, accounting for $17.90 \%$ (summer sample) and $8.25 \%$ (winter sam- ple) at the outdoor location. However, the proportion was higher than the indoor samples at the same season, probably because the outdoor air with higher flow was influenced by temperature, humidity, air currents, and other environmental factors. In addition, some of the pathogens collected from the PIF production environment (Micrococcus, Pseudomonas, and Kocuria) were found in various environments such as smog days and the ocean (Fahlgren et al., 2010; Martin et al., 2010; Cao et al., 2014). The distribution of genera at different sampling locations is shown in Supplemental Tables S2 and S3 (https://doi.org/10.3168/jds.2017-13968).

Overall, the bacterial community structure in the air of the PIF factory varied according to the different methods. The traditional cultivation-based method has previously been considered to be a gold standard for monitoring airborne bacteria (Hubad and Lapanje, 2013), although a limitation of the method is the requirement that the bacteria be culturable. Due to inappropriate culturing conditions or medium type, many important hard-to-culture species can be overlooked and difficult to identify accurately. It might lead to the underestimation of the true bacterial profile (McHugh et al., 2017). In addition, a total of 1,062 TSA plates 
were collected in this study. The sampling method for the culturable organisms was time consuming and labor intensive. In contrast, for high-throughput sequencing, there was no need to culture bacteria and the number of air samples was only 12 - considerably less than the cultivation method. The high-throughput sequencing method revealed previously undetectable microbial diversity as well as the more comprehensive microbial communities in the air of PIF processing environments. For example, some uncultured bacteria were found in the processing environment of PIF, such as Anaerolineaceae (Supplemental Tables S2 and S3; https://doi.org/10.3168/jds.2017-13968). Therefore, the high-throughput method can be coupled with the cultivation-based method to obtain an overview of culturable and unculturable airborne bacteria in the different processing areas. The key processes generating airborne bacteria can be tracked and identified to reduce the possibility of unknown or underestimated microbiology-related food safety threats in the production environment of PIF (McHugh et al., 2017).

Seasonal Distribution of Bacteria at the Genus Level. In this study, air samples were collected from 2 different seasons to explore the differences of microbial community structures. The prevalent strains and the bacterial community structures of air samples varied significantly with seasonal variations. In the winter samples, Lactococcus was the dominant genus, with an average relative proportion of $45.69 \%$, followed by Staphylococcus (27.70\%), Bacillus (14.98\%), and Lysinibacillus (2.58\%). In the summer samples, Bacillus (44.12\%) was the dominant genus, followed by Lactococcus (18.98\%), Pseudomonas (11.38\%), and Gammaproteobacteria unclassified (5.81\%).

Comparative analysis of the airborne bacterial community structures during winter and summer sampling revealed distinct distributions of bacteria in the PIF production environment. The relative abundances of some genera changed in different seasons. For instance, Staphylococcus was observed with low percentages $(<1.05 \%)$ in summer samples but accounted for more than $25.65 \%$ of the total communities in winter samples. Bacillus, Gammaproteobacteria unclassified, and Psychrobacter had lower relative abundances in summer than in winter. However, Lactococcus showed the opposite trend (Figure 2). Among the 6 sampling sites, Bacillus was present in the highest proportion in the winter outdoor sampling site, accounting for $17.58 \%$ of the total bacterial communities, whereas in summer for the outdoor site it was present in the smallest proportion $(39.02 \%)$; the site with the highest relative abundance of Bacillus (47.33\%) during summer sampling was the large bag filling process area. In all sampling sites, the relative abundances of other genera from summer samples were much higher than those from winter samples. The phenomenon likely was due to the bacterial community with higher diversity during summer sampling. This result was also consistent with the differences $(P<0.05)$ shown in Table 2 .

The principal component analysis of different samples based on OTU at a distance of $3 \%$ was used to analyze the similarity and difference of bacterial community structures among 6 sampling locations in winter and summer (Figure 3). The results revealed that all air samples were clustered into 2 groups. Six samples collected from the winter were classified into group 1, and group 2 contained 6 samples collected from the summer. This cluster indicated that bacterial community structures at the different sampling locations were similar for the same season, but it was unclear to divide air samples into different groups according to the variation of sampling locations. The results of principal component analyses were in accordance with the microbial community structure and distribution discovered from winter and summer samples.

Environmental factors (climate, temperature, relative humidity, and air velocity) could affect the number and concentrations of bacteria and the bacterial microbial community structure and distribution (Huang et al., 2002; Jones and Harrison, 2004; Wang et al.,

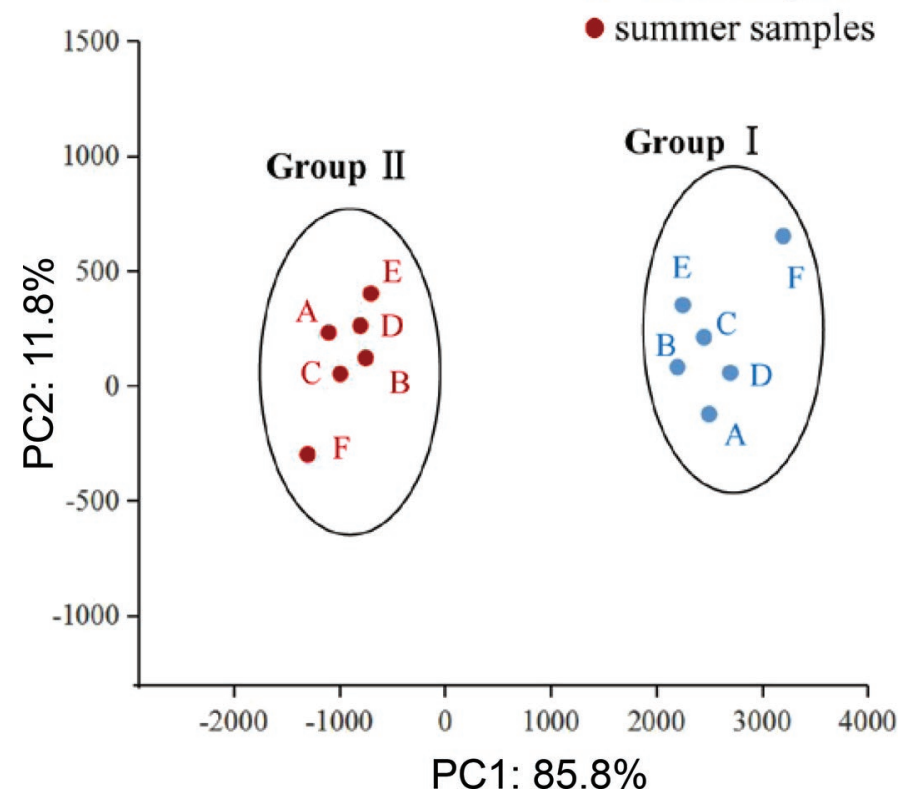

Figure 3. Principal component (PC) analysis of air samples based on the structures of the microbial community. Indoor sampling locations covered the whole processing line, including pretreatment (A), production (B), large bag filling (C), can filling (D), and packaging (E). An outdoor sampling location (F) was used as the reference. Color version available online. 
2010). In particular, climate variation with different seasons is an important factor influencing the composition of airborne microorganisms. In our study, at the same sampling site, the distribution of bacterial genera exhibited obvious differences during different sampling seasons. The most likely reason was that higher temperature, humidity, and other factors such as personnel movement in the factory in summer could promote the higher airborne bacterial content and increase microbial diversity compared with winter in the PIF production environment.

In the processing of dairy products, general handling of ingredients, spray drying, and wet and dry cleaning operations can create bioaerosols, with various bacteria being attached (Mullane et al., 2008; Dungan, 2012). It is impossible to keep airborne bacteria at a zero level in food processing. Textile filters for exhaust air of spray-drying towers have been identified as a reservoir for pathogens during the milk powder production. The final product can be contaminated with the powder from such textile filters (Jacobs et al., 2011). Thus, air quality should be routinely monitored, especially the areas that require high hygienic levels. Air filters of vacuum cleaners could be used for air exhaust to maintain good hygiene in the processing environment.

Food hygiene is essential for food processing, including the hygienic design and engineering of facilities and equipment and integration of components and maintenance (Betta et al., 2011). Good hygienic levels in the processing environment can be ensured and maintained by the implementation of food safety management systems, such as good manufacturing practice, standard sanitation operating procedures, and hazard analysis and critical control points (Carrascosa et al., 2016). Moreover, it is vital to reduce the contamination of airborne bacteria during hygienic processing. However, lack of expertise, financial constraints, attitudes, and other legal requirements are potential difficulties in food safety processing (Garayoa et al., 2011). In our study, the staff members could be the potential carriers of bacteria in the PIF factory. Hence, unnecessary human activities should be reduced in the production, packaging, and filling areas. The production facility personnel must be aware of the importance of food safety management systems and improve their knowledge related to good hygienic practices. Importantly, standard sanitary operating procedures cannot be replaced by the use of antimicrobial additives (Obaidat et al., 2015). For example, the implementation of food safety management systems at a yogurt production plant necessitated structural changes and the purchase of new equipment, which together accounted for $83.75 \%$ of the total cost of the plant. Subsequently, the reduction in microbial contamination and an increase of shelf life contributed to the reduction of the overall costs for production (Cusato et al., 2013; Oliveira et al., 2014). Thus, food safety systems must be strictly implemented to maintain a good hygienic processing environment. Our study could aid food safety processing to reduce the potential airborne microbial risks and contamination rates of $\mathrm{PIF}$.

\section{CONCLUSIONS}

In summary, this study revealed the microbial community structure and distribution of different sampling sites in the PIF factory by cultivation-based and Illumina high-throughput methods. A total of 140 bacterial OTU and 104 genera obtained from air samples indicated that Illumina sequencing could accelerate the discovery of the bacterial community in the PIF production environment. Additionally, the air in a PIF factory may be a potential route for the transmission of airborne bacteria to key processing areas. Therefore, our study can provide a guide for reducing the risk of microbial contamination in the production of PIF.

\section{ACKNOWLEDGMENTS}

This work was supported by the "Academic Backbone" Project of Northeast Agricultural University (Harbin, China; 15XG26).

\section{REFERENCES}

Abdullah Sani, N., S. H. Hartantyo, and S. J. Forsythe. 2013. Microbiological assessment and evaluation of rehydration instructions on powdered infant formulas, follow-up formulas, and infant foods in Malaysia. J. Dairy Sci. 96:1-8.

Angulo, F. J., S. M. Cahill, I. K. Wachsmuth, M. de Lourdes Costarrica, and P. K. B. Embarek. 2008. Powdered infant formula as a source of Salmonella infection in infants. Clin. Infect. Dis. 46:268273.

Araújo, B. C., M. S. Moraes, L. E. Costa, and J. S. Nascimento. 2015. Short communication: Multidrug-resistant Acinetobacter baumannii-calcoaceticus complex isolated from infant milk formula and utensils in a nursery in Rio de Janeiro, Brazil. J. Dairy Sci. 98:2303-2306.

Stenfors Arnesen, L. P., A. Fagerlund, and P. E. Granum. 2008. From soil to gut: Bacillus cereus and its food poisoning toxins. FEMS Microbiol. Rev. 32:579-606.

Betta, G., D. Barbanti, and R. Massini. 2011. Food hygiene in aseptic processing and packaging system: A survey in the Italian food industry. Trends Food Sci. Technol. 6:327-334.

Brandl, H., C. Fricker-Feer, D. Ziegler, J. Mandal, R. Stephan, and A. Lehner. 2014. Distribution and identification of culturable airborne microorganisms in a Swiss milk processing facility. J. Dairy Sci. 97:240-246.

Cao, C., W. Jiang, B. Wang, J. Fang, J. Lang, G. Tian, J. Jiang, and T. F. Zhu. 2014. Inhalable microorganisms in Beijing's PM 2.5 and PM 10 pollutants during a severe smog event. Environ. Sci. Technol. 48:1499-1507. 
Carrascosa, C., R. Millã, P. Saavedra, J. R. Jaber, A. Raposo, and E. Sanjuã. 2016. Identification of the risk factors associated with cheese production to implement the hazard analysis and critical control points (HACCP) system on cheese farms. J. Dairy Sci. 99:2606-2616.

Chao, A. 1984. Nonparametric estimation of the number of classes in a population. Scand. J. Stat. 11:265-270.

Craven, H. M., C. M. McAuley, L. L. Duffy, and N. Fegan. 2010. Distribution, prevalence and persistence of Cronobacter (Enterobacter sakazakii) in the nonprocessing and processing environments of five milk powder factories. J. Appl. Microbiol. 109:1044-1052.

Cusato, S., A. H. Gameiro, C. H. Corassin, A. S. Sant'ana, A. G. Cruz, J. A. Faria, and C. A. de Oliveira. 2013. Food safety systems in a small dairy factory: Implementation, major challenges, and assessment of systems' performances. Foodborne Pathog. Dis. 10:6-12.

Dungan, R. S. 2012. Use of culture-independent approach to characterize aerosolized bacteria near open-freestall dairy operation. Environ. Int. 41:8-14.

Eckburg, P. B., E. M. Bik, C. N. Bernstein, E. Purdom, L. Dethlefsen, M. Sargent, S. R. Gill, K. E. Nelson, and D. A. Relman. 2005. Diversity of the human intestinal microbial flora. Science 308:1635-1638.

Eduard, W., D. Heederik, C. Duchaine, and B. J. Green. 2012. Bioaerosol exposure assessment in the workplace: The past, present and recent advances. J. Environ. Monit. 14:334-339.

Er, B., B. Demirhan, and G. Yentür. 2014. Short communication: Investigation of aflatoxin $\mathrm{M}_{1}$ levels in infant follow-on milks and infant formulas sold in the markets of Ankara, Turkey. J. Dairy Sci. 97:3328-3331.

Fahlgren, C., A. Hagström, D. Nilsson, and U. L. Zweifel. 2010. Annual variations in the diversity, viability, and origin of airborne bacteria. Appl. Environ. Microbiol. 76:3015-3025.

FAO/WHO (Food and Agriculture Organization/World Health Organization). 2004. Enterobacter sakazakii and other microorganisms in powdered infant formula: Meeting report. Microbiological Risk Assessment Series 6. FAO/WHO, Geneva, Switzerland.

Fei, P., C. Man, B. Lou, S. J. Forsythe, Y. Chai, R. Li, J. Niu, and Y. Jiang. 2015. Genotyping and source tracking of Cronobacter sakazakii and C. malonaticus isolates from powdered infant formula and an infant formula production factory in China. Appl. Environ. Microbiol. 81:5430-5439.

Fricker-Feer, C., N. Cernela, S. Bolzan, A. Lehner, and R. Stephan. 2011. Evaluation of three commercially available real-time PCR based systems for detection of Cronobacter species. Int. J. Food Microbiol. 146:200-202.

Garayoa, R., A. I. Vitas, M. Díez-Leturia, and I. García-Jalón. 2011. Food safety and the contract catering companies: Food handlers, facilities and HACCP evaluation. Food Control 22:2006-2012.

Guo, X., P. Wu, W. Ding, W. Zhang, and L. Li. 2014. Reduction and characterization of bioaerosols in a wastewater treatment station via ventilation. J. Environ. Sci. (China) 26:1575-1583.

Horner-Devine, M. C., K. M. Carney, and B. J. Bohannan. 2004. An ecological perspective on bacterial biodiversity. Proc. Biol. Sci. 271:113-122.

Hu, M., X. Wang, X. Wen, and Y. Xia. 2012. Microbial community structures in different wastewater treatment plants as revealed by 454-pyrosequencing analysis. Bioresour. Technol. 117:72-79.

Huang, C.-Y., C.-C. Lee, F.-C. Li, Y.-P. Ma, and H.-J. J. Su. 2002. The seasonal distribution of bioaerosols in municipal landfill sites: A 3-yr study. Atmos. Environ. 36:4385-4395.

Hubad, B., and A. Lapanje. 2013. The efficient method for simultaneous monitoring of the culturable as well as nonculturable airborne microorganisms. PLoS One 8:e82186.

Iversen, C., and S. Forsythe. 2004. Isolation of Enterobacter sakazakii and other Enterobacteriaceae from powdered infant formula milk and related products. Food Microbiol. 21:771-777.

Jacobs, C., P. Braun, and P. Hammer. 2011. Reservoir and routes of transmission of Enterobacter sakazakii (Cronobacter spp.) in a milk powder-producing plant. J. Dairy Sci. 94:3801-3810.
Jones, A. M., and R. M. Harrison. 2004. The effects of meteorological factors on atmospheric bioaerosol concentrations-A review. Sci. Total Environ. 326:151-180.

Juma, N. A., G. Manning, and S. J. Forsythe. 2016. Desiccation survival of Acinetobacter spp. in infant formula. Food Control 68:162166.

Kabak, B. 2012. Aflatoxin $\mathrm{M}_{1}$ and ochratoxin $\mathrm{A}$ in baby formulae in Turkey: Occurrence and safety evaluation. Food Control 26:182187.

Kent, R. M., G. F. Fitzgerald, C. Hill, C. Stanton, and R. P. Ross. 2015. Novel approaches to improve the intrinsic microbiological safety of powdered infant milk formula. Nutrients 7:1217-1244.

Lee, B. U. 2011. Life comes from air: A short review on bioaerosol control. Aerosol Air Qual. Res. 11:921-927.

Leung, M. H., D. Wilkins, E. K. Li, F. K. Kong, and P. K. Lee. 2014. Indoor-air microbiome in an urban subway network: Diversity and dynamics. Appl. Environ. Microbiol. 80:6760-6770.

Li, J., L. Zhou, X. Zhang, C. Xu, L. Dong, and M. Yao. 2015. Bioaerosol emissions and detection of airborne antibiotic resistance genes from a wastewater treatment plant. Atmos. Environ. 124:404-412.

Lu, Y., Y. Chen, X. A. Lu, J. Lv, C. X. Man, Y. L. Chai, and Y. J. Jiang. 2014. Comparison of methods for the microbiological identification and typing of Cronobacter species in infant formula. J. Dairy Sci. 97:632-641.

Martin, E., P. Kämpfer, and U. Jäckel. 2010. Quantification and identification of culturable airborne bacteria from duck houses. Ann. Occup. Hyg. 54:217-227.

McHugh, A. J., C. Feehily, C. Hill, and P. D. Cotter. 2017. Detection and enumeration of spore-forming bacteria in powdered dairy products. Front. Microbiol. 8:109.

Miao, Y., R. Liao, X.-X. Zhang, Y. Wang, Z. Wang, P. Shi, B. Liu, and A. Li. 2015. Metagenomic insights into Cr (VI) effect on microbial communities and functional genes of an expanded granular sludge bed reactor treating high-nitrate wastewater. Water Res. 76:43-52.

Michaelsen, A., G. Piñar, and F. Pinzari. 2010. Molecular and microscopical investigation of the microflora inhabiting a deteriorated Italian manuscript dated from the thirteenth century. Microb. Ecol. 60:69-80.

Mullane, N., B. Healy, J. Meade, P. Whyte, P. Wall, and S. Fanning. 2008. Dissemination of Cronobacter spp. (Enterobacter sakazakii) in a powdered milk protein manufacturing facility. Appl. Environ. Microbiol. 74:5913-5917.

Mullane, N. R., C. Iversen, B. Healy, C. Walsh, P. Whyte, P. Wall, T. Quinn, and S. Fanning. 2007. Enterobacter sakazakii an emerging bacterial pathogen with implications for infant health. Minerva Pediatr. 59:137-148.

Obaidat, M. M., M. H. Alu'Datt, A. E. B. Salman, H. M. Obaidat, A. A. Al-Zyoud, O. K. Al-Saleh, and B. A. Al'Anaz. 2015. Inactivation of nondesiccated and desiccated Cronobacter sakazakii and Salmonella spp. at low and high inocula levels in reconstituted infant milk formula by vanillin. Food Control 50:850-857.

Oliveira, C. A. F. D., S. Cusato, A. D. S. Sant'Ana, A. G. D. Cruz, and C. H. Corassin. 2014. Assessing the costs involved in the implementation of GMP and HACCP in a small dairy factory. Qual. Assur. Saf. Crops Foods 6:135-139.

Poulsen, P. H., W. A. Al-Soud, L. Bergmark, J. Magid, L. H. Hansen, and S. J. Sereneness. 2013. Effects of fertilization with urban and agricultural organic wastes in a field trial-Prokaryotic diversity investigated by pyrosequencing. Soil Biol. Biochem. 57:784-793.

Proudy, I., D. Bougle, R. Leclercq, and M. Vergnaud. 2008. Tracing of Enterobacter sakazakii isolates in infant milk formula processing by BOX-PCR genotyping. J. Appl. Microbiol. 105:550-558.

R Core Team. 2015. R: A language and environment for statistical computing. Computing 1:12-21.

Rachon, G., W. Peñaloza, P. Breeuwer, J. Guan, A. Knight, I. Campelos, and P. A. Gibbs. 2017. Poor hygiene practices in infant formulae reconstitution and inappropriate storage of feeding bottles can cause spoilage issues by Serratia marcescens. Food Control 79:150-155. 
Riesenfeld, C. S., P. D. Schloss, and J. Handelsman. 2004. Metagenomics: Genomic analysis of microbial communities. Annu. Rev. Genet. 38:525-552.

Roesch, L. F., R. R. Fulthorpe, A. Riva, G. Casella, A. K. Hadwin, A. D. Kent, S. H. Daroub, F. A. Camargo, W. G. Farmerie, and E W. Triplett. 2007. Pyrosequencing enumerates and contrasts soil microbial diversity. ISME J. 1:283-290.

Schloss, P. D., S. L. Westcott, T. Ryabin, J. R. Hall, M. Hartmann E. B. Hollister, R. A. Lesniewski, B. B. Oakley, D. H. Parks, and C. J. Robinson. 2009. Introducing mothur: Open-source, platformindependent, community-supported software for describing and comparing microbial communities. Appl. Environ. Microbiol. 75:7537-7541

Sonbol, H., S. Joseph, C. McAuley, H. Craven, and S. J. Forsythe. 2013. Multilocus sequence typing of Cronobacter spp. from powdered infant formula and milk powder production factories. Int. Dairy J. 30:1-7.

Stetzenbach, L. D., M. P. Buttner, and P. Cruz. 2004. Detection and enumeration of airborne biocontaminants. Curr. Opin. Biotechnol. 15:170-174.

Tian, W., Z. Zhang, X. Hu, R. Tian, J. Zhang, X. Xiao, and Y. Xi 2015. Short-term changes in total heavy metal concentration and bacterial community composition after replicated and heavy application of pig manure-based compost in an organic vegetable production system. Biol. Fertil. Soils 51:593-603.

Wang, Q., G. M. Garrity, J. M. Tiedje, and J. R. Cole. 2007. Naive Bayesian classifier for rapid assignment of rRNA sequences into the new bacterial taxonomy. Appl. Environ. Microbiol. 73:5261-5267.
Wang, W., Y. Ma, X. Ma, F. Wu, X. Ma, L. An, and H. Feng. 2010 Seasonal variations of airborne bacteria in the Mogao Grottoes, Dunhuang, China. Int. Biodeterioration Biodegradation 64:309315.

Washington, H. 1984. Diversity, biotic and similarity indices: A review with special relevance to aquatic ecosystems. Water Res. 18:653-694

Xu, F., P. Li, X. Ming, D. Yang, H. Xu, X. Wu, N. P. Shah, and H. Wei. 2014. Detection of Cronobacter species in powdered infant formula by probe-magnetic separation PCR. J. Dairy Sci. 97:60676075.

Yan, Q. Q., O. Condell, K. Power, F. Butler, B. Tall, and S. Fanning. 2012. Cronobacter species (formerly known as Enterobacter sakazakii) in powdered infant formula: A review of our current understanding of the biology of this bacterium. J. Appl. Microbiol. 113:1-15.

Zhang, X.-Y., G.-H. Wang, X.-Y. Xu, X.-H. Nong, J. Wang, M. Amin and S.-H. Qi. 2016. Exploring fungal diversity in deep-sea sediments from Okinawa Trough using high-throughput Illumina sequencing. Deep Sea Res. Part I Oceanogr. Res. Pap. 116:99-105.

Zollinger, M., W. Krebs, and H. Brandl. 2006. Bioaerosol generation during grape stemming and crushing. Sci. Total Environ. 363:253259. 